\title{
Influence of the Angular Momentum in Problems Continuum Mechanics
}

\author{
EVELINA PROZOROVA \\ Mathematical-Mechanical Department \\ St. Petersburg State University \\ Av. 28 , Peterhof, 198504 \\ RUSSIA
}

\begin{abstract}
For continuum mechanics a model is proposed, that is built with consideration outside the integral term when deriving conservation laws using the Ostrogradsky-Gauss theorem. Performed analysis shows discrepancy between accepted classical conservation laws and classical theoretical mechanics and mathematics. As a result, the theory developed for potential flows was extended to flows with significant gradients of physical parameters. We have proposed a model that takes into account the joint implementation of the laws for balance of forces and angular momentums. It does not follow from the Boltzmann equation that the pressure in the Euler and Navier-Stokes equations is equal to one third of the sum the pressures on the corresponding coordinate axes. The vector definition of pressure is substantiated. It is shown that the symmetry condition for the stress tensor is one of the possible conditions for closing the problem. An example of solving the problem of the theory of elasticity is given.
\end{abstract}

Keywords: angular moment, potential flow, circulation, Euler equation, Pascal's law

Received: September 26, 2020. Revised: February 13, 2021. Accepted: February 26, 2021. Published: March 4, 2021.

\section{Introduction}

In aeromechanics, shipbuilding, the physics of earth and atmosphere, there are no understanding of some processes associated with the interaction of flows with body which are moving at high speeds; there are no adequate models for the formation of vortex structures, etc. The objects of study in continuum mechanics are solids, liquid, gas, and plasma. In a mathematical description, the basic laws are conservation laws. This unites them. Therefore, the work discusses general questions arising when writing conservation laws. The law of equilibrium of forces was put as basis of the classical theory, the law of conservation angular momentum was considered as consequence of the fulfillment the law of balance forces. Each of laws was considered independently. However, the gradient of the distributed moment leads to the formation of an additional force, that we need to take into account in the equations of motion. This is evidenced by the works devoted to the calculation of stresses in the rods [1,2] and in the calculations of viscoelastic problems [3]. The probable reason for ignoring the effect in the general theory is the proof of its absence, built on the basis of the theory, taking into account the interaction of neighboring elementary volumes only along the normal to the surface [4-6]. In this case, one can only prove the consistency of the whole theory. The influence of the rotational part of the stress tensor can be traced by analyzing the Hamel solution and its generalizations [7]. Dividing the speed into divergent and rotational components about an axis passing through an arbitrary point does not right, as according to theory, only movement around the axis of inertia need to consider. A model that is ignoring the rotation of the elementary volume leads to the symmetry of the stress tensor, which violates the "continuity" of the medium [8]. When analyzing the derivation of differential conservation laws in continuum mechanics, it turned out that the transition 
from the integral formulation to the differential formulation (integration by parts) was performed without taking into account outside integral component. In classical version, the Ostrogradsky-Gauss theorem is formulated for a motionless body without rotation [9]. Thus, the generalization of the theorem to body that is rotating [10] leads to a stronger influence of the no symmetry of the stress tensor in the entire mechanics of a continuous medium. After integration by parts, the transition from the integral formulation to the differential formulation is difficult. However, one can take advantage of physical and geometric considerations and derive equations from them. At present, the need to divide the stress tensor into two parts is not clear. The speeds of various processes at the time of writing the equations were relatively low compared to modern one. Subsequently, the theory that was developed for potential flows was used to flows with significant gradients of physical parameters was expanded. The lack of symmetry leads to an increase in the order of the derivatives the Navier-Stokes equations. The role of boundary conditions increases accordingly. Their specific form is determined by the problem under consideration. Since the original formulation obtained in experiments is integral, then numerical methods using conservation laws in integral form are preferable. These methods include the finite volume method. The implementation of the method is achieved in various ways. Equations of an incompressible fluid are best approximated. For a compressible fluid, the computational process becomes more complicated. It is important to use the integral conservation laws obtained from the experiment. The transition from the currently used equations to integral ones due to the rejection to investigate of the rotation effects does not allow the conservation laws to be fulfilled. The experimentally confirmed Newton's law on the relationship between the stress and strain tensor concerned the total velocity with the no symmetric tensor. In the kinetic theory, the coefficients of viscosity and thermal conductivity, even in no equilibrium version, use only the equilibrium part of the Chapman-Enskog function, the rest of the terms included in the function do not contribute. Consequently, the full account of the viscous contribution is ensured by the full velocity, in fact, by its corresponding first derivatives. However, the macroparameters included in the equilibrium distribution function must be calculated not according to zero approximation from the Euler equations, but from the equations of the first approximation (the Navier-Stokes equations, if they are used). The equations of motion obtained from the Boltzmann equation correspond to the zero order for the Euler equations and the first order for the Navier-Stokes equations. Using only divergent part of velocity without rotation it we obtain speed different from the initial one. The situation is saved by the fact that this circumstance affects the viscous component of stresses, which is a first-order quantity. Hydrostatic pressure is a zeroorder quantity. Using Pascal's law for equilibrium, the pressure is chosen equal to one third of the pressure on the coordinate areas. It does not follow from the Boltzmann equation that the pressure in the Euler equation is equal to one third of the sum of the pressures on the corresponding coordinate axes. There is no experimental confirmation of this fact. However, the theory remains the same when determining the different pressure on each of the sites, i.e. the use of one pressure is possible under equilibrium conditions (Pascal's law), but for no equilibrium conditions the fact is not obvious. Attention is drawn to this in the textbook [11] and articles [12,13]. In the theory of elasticity, when considering the relationship between the components of the deformation tensor and the stress tensor, the experimental fact of the change in the components (stresses) normal to the elementary site is used in proportion to the sum of the stresses from others components, and they all differ. If problems of the theory of elasticity was solving with a symmetric tensor, it is necessary to satisfy the compatibility conditions $[5,6]$. To get around these conditions when solving the problem numerically, the function values in 
the cell are "averaged". The result of the calculation is then the values in the center. This is not discussed in the articles, but in private conversations the fact is admitted. Experimental fact of the no symmetry of the stress tensor can be found in the works $[14,15]$, theory in [16]. The existing theory is connected with the fact that the derivation of conservation laws in the theory of elasticity excludes the contribution of the distributed moment to the conditions of equilibrium of forces. An interesting feature of the equations of hydrodynamics is the absence of nesting of models equations into each other. For example, the potential flow cannot be obtained from the Euler equations. Static pressure, as follows from the kinetic theory, is a zero-order quantity, and the terms associated with dissipative effects are first-order quantities. As already noted, it does not follow from the Boltzmann equation that the pressure in the Euler equation is equal to one third of the sum of the pressures on the corresponding coordinate axes. Inaccuracy in determining the velocities in the stress tensor does not greatly affect the results. For an incompressible fluid, the speeds are the same. The most important characteristic of an elementary volume is its position of the center of inertia and the correct projection of the boundaries of an arbitrary volume on the coordinate axes. Due to the arbitrariness of the directions of motion, the viscous components of the velocity must be taken into account even in one-dimensional motion. With any restructuring of the flow, the position of the center of inertia changes, a moment of force is created, the gradient of which is the force itself. Additional force is added with stresses and change the direction of movement and the speed of the molecules. The result is a change in the equation of state even under equilibrium conditions and the formation of fluctuation phenomena. The equation of state (Newton's law) in classical mechanics ensures the symmetry of the stress tensor. Note again that the classical equations do not include all the speeds that need to be considered. The vortex part is discarded. The experiment does not separate speeds, measuring the total contribution to friction and pressure. Measurements performed on the axes of symmetry do not allow speaking about the degree of asymmetry of the stress tensor. The existence of invariants higher than the first and, accordingly, the principal axes of the elementary volume in the proposed theory of elasticity becomes problematic. The question of equilibrium in mechanics of a continuous medium and the extension of Pascal's law to the case of small deformations, the need to replace the averaged pressure with its theoretical values $p_{x x}, p_{y y}, p_{z z}$ are discussed. The rejection of pressure averaging makes it possible to explain the formation of vortex bundles when flowing around bodies in the vicinity of the separation point. It is very difficult to judge the influence of the moment, limiting ourselves to the results of calculating twodimensional problems. If the tensor is asymmetric, then we have three equations for four unknowns. The system is not closed. Therefore, additional assumptions have to be made. One of these assumptions is the assumption about the symmetry of the stress tensor. These issues are discussed in this work.

\section{Origin of the angular momentum}

Consider the interaction of three particles

interacting with each other. Since the angular momentum is equal to the force acting on the center of inertia, that is vector multiplied by the radius vector, we can calculate the changes in the moment after a certain period of time

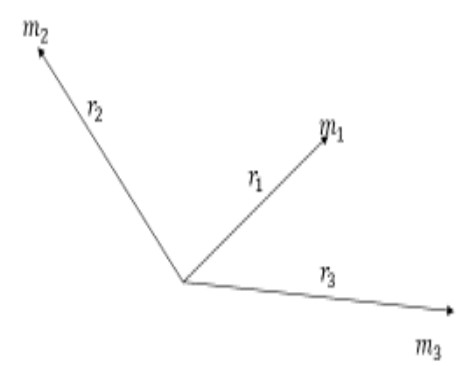

Fig. 1. Interaction of three particles

$r_{c}=\frac{m_{1} r_{1}+m_{2} r_{2}+m_{3} r_{3}}{m_{1}+m_{2}+m_{3}}$, 


$$
r_{c+\Delta c}=\frac{m_{1}\left(r_{1}+\dot{r_{1}} \Delta t\right)+m_{2}\left(r_{2}+\dot{r_{2}} \Delta t\right)+m_{3}\left(r_{3}+\dot{r_{3}} \Delta t\right)}{m_{1}+m_{2}+m_{3}}
$$

At the next moment in time, the center of inertia will shift. Therefore, an additional force arises, since the gradient of the moment is a force. Thus, the moment creates a collective force for any movement of particles with different speeds. The effect works in the formation of fluctuations and must be taken into account when calculating the equation of state. In continuum mechanics, the integral conservation laws obtained experimentally are written in differential form.

When passing to differential equations, the Ostrogradsky-Gauss theorem is used for the selected fixed volume, ignoring the possible rotation of the elementary volume. The discarded term represents the velocity circulation, its rotor part. Circulation is the action of the moment. Consequently, the rotational terms (moment) discarded in the construction of the Navier-Stokes equations are formed not only in the case of large gradients, but also due to the additional circulation of the velocity around the elementary volume [10].

The influence of the moment is also manifested through pressure. There are no experiments confirming Pascale's law in non-equilibrium cases. The kinetic theory (modified and classical) also does not follow Pascal's law.

Consider the classical Boltzmann equation (the law of momentum does not hold)

$$
\begin{gathered}
\mathbf{u}(t, \mathbf{x})=\frac{1}{n} \int \xi f(t, \mathbf{x}, \boldsymbol{\xi}) d \xi, \\
P_{i j}=m \int c_{j} c_{i} f(t, \mathbf{x}, \boldsymbol{\xi}) d \boldsymbol{\xi}, \\
q_{;}=m \int c^{2} c_{i} f(t, \mathbf{x}, \boldsymbol{\xi}) d \xi, \\
\boldsymbol{c}=\boldsymbol{\xi}-\mathbf{u} . \\
f\left(t+d t, \boldsymbol{r}+\boldsymbol{\xi}_{j} d t, \boldsymbol{\xi}_{j}+\boldsymbol{F}_{j} d t\right) d r d \xi_{j}= \\
f\left(\boldsymbol{r}, \xi_{j}, \mathrm{t}\right) d \boldsymbol{r} d \xi_{j}+\left(\frac{\partial f}{\partial t}\right)_{c o l l} d t .
\end{gathered}
$$

$f$ distribution function, $r$ - radius vector; $x$ point coordinate; $\xi$ is the velocity of a point, is the molecular weight, and, according to the definition of the distribution function $f_{N}$, the probability of finding the system at points $(x, \xi)$ in the intervals $\boldsymbol{d} x_{\boldsymbol{i}} \boldsymbol{d} \xi_{\boldsymbol{i}}$ is
$f_{N}\left(t, x_{1}, x_{2}, \ldots x_{N}, \xi_{1}, \xi_{2}, \ldots \xi_{N}\right) d x_{1} \ldots d x_{N} d \xi_{1} \ldots d \xi_{N}$ . To construct an equation directly for the velocity projection, we multiply the equation by the velocity projection $\xi$ on the coordinate axis, for example, $x$. Let us have different pressure values $p_{1}, p_{2}, p_{3}$ :

$$
\begin{aligned}
M_{x} & =y p_{3}-z, \\
M_{Y} & =-x p_{3}+z p_{1}, \\
M_{z} & =x p_{2}-y p_{1} .
\end{aligned}
$$

Really,

$$
\left[\begin{array}{ccc}
i & j & k \\
x & y & z \\
p_{1} & p_{2} & p_{3}
\end{array}\right]
$$

Consequently, the impossibility of the same values of pressure $\mathrm{p}$. Pressure is a vector like force. In this case, the Lamb equation [7] has the form

$$
\begin{aligned}
& \frac{\partial V}{\partial t}+\operatorname{grad}\left(\frac{V^{2}}{2}\right)+\operatorname{rot} V \times V=F- \\
& \frac{1}{\rho} \operatorname{grad} \vec{P}
\end{aligned}
$$

and there is a dependence of density as an average value over an elementary volume, then the Bernoulli equation makes sense. The finite volume method is often used to numerically solve the Euler and NavierStokes equations. However, its formulation is based on differential equations with a symmetric stress tensor. In the mechanics of liquid and gas, it is not customary to average the calculation results over the calculated cells. Consequently, since the mesh has finite dimensions, in old works, the no symmetry of the tensor is partially taken into account in numerical calculations. In solid mechanics, averaging is offer performed when solving problems by the finite element method. With a large number of steps, this gives significant errors. The main argument for choosing a symmetric tensor is the use of the equilibrium equation for the force without the influence of the moment. However, the momentum gradient is force. Consequently, the requirement of simultaneous fulfillment of the laws of balance of forces and moments of forces changes the equations.

In general case 


$$
\begin{aligned}
& \rho\left(\frac{\partial u}{\partial t}+u \frac{\partial u}{\partial x}+v \frac{\partial u}{\partial y}+w \frac{\partial u}{\partial z}\right)=\rho f_{1} \\
& +\frac{\partial \sigma_{x x}}{\partial x}+\frac{\partial \sigma_{y x}}{\partial y}+\frac{\partial \sigma_{z x}}{\partial z}+\rho f_{M_{x}} \\
& \rho\left(\frac{\partial v}{\partial t}+u \frac{\partial v}{\partial x}+v \frac{\partial v}{\partial y}+w \frac{\partial v}{\partial z}\right)= \\
& \rho f_{2}+\frac{\partial \sigma_{x y}}{\partial x}+\frac{\partial \sigma_{y y}}{\partial y}+\frac{\partial \sigma_{z y}}{\partial z}+\rho f_{M_{y}} \\
& \rho\left(\frac{\partial w}{\partial t}+u \frac{\partial w}{\partial x}+v \frac{\partial w}{\partial y}+w \frac{\partial w}{\partial z}\right)= \\
& \rho f_{3}+\frac{\partial \sigma}{\partial x}+\frac{\partial P \sigma_{y z}}{\partial y}+\frac{\partial \sigma_{z z}}{\partial z}+\rho f_{M_{z}} \\
& y\left(\frac{\partial \sigma_{x z}}{\partial x}+\frac{\partial \sigma_{y z}}{\partial y}+\frac{\partial \sigma_{z z}}{\partial z}+\rho f_{3}\right)- \\
& z\left(\frac{\partial \sigma_{x y}}{\partial x}+\frac{\partial \sigma_{y y}}{\partial y}+\frac{\partial \sigma_{z y}}{\partial z}+\rho f_{2}\right)+ \\
& \sigma_{z y}-\sigma_{z y}++M_{x}=0 \\
& x\left(\frac{\partial \sigma_{x y}}{\partial x}+\frac{\partial \sigma_{y y}}{\partial y}+\frac{\partial \sigma_{z y}}{\partial z}+\rho f_{2}\right)- \\
& y\left(\frac{\partial \sigma_{x x}}{\partial x}+\frac{\partial \sigma_{y x}}{\partial y}+\frac{\partial \sigma_{z x}}{\partial z}+\rho f_{1}\right)+ \\
& \sigma_{y x}-\sigma_{x y}++M_{y}=0 \\
& x\left(\frac{\partial \sigma_{x z}}{\partial x}+\frac{\partial \sigma_{y z}}{\partial y}+\frac{\partial \sigma_{z z}}{\partial z}+\rho f_{1}\right)- \\
& z\left(\frac{\partial \sigma_{x x}}{\partial x}+\frac{\partial \sigma_{y x}}{\partial y}+\frac{\partial \sigma_{z x}}{\partial z}+\rho f_{2}\right)+ \\
& \sigma_{z x}-\sigma_{x z}++M_{z}=0
\end{aligned}
$$

Here

all

designations

are standard, $f_{M_{x}}, f_{M_{y}}, f_{M_{z}}$ forces created by the moment, $M_{x}, M_{y}, M_{z}$ are external moments.

\section{Specific tasks}

In the three-dimensional case, we have six unknowns and six equations for the no symmetric tensor, but used only three. In the flat version, there is one equation for the moments and two for movement, unknown four. Closing can be by the symmetry condition of the stress tensor. But you can do it in another way. Some problems with symmetric tensor cannot be solved. Let us consider the problem when the stress distributions are given, with and without taking into account the influence of the angular momentum. The voltages can be the same, they can be different. Considered case of elongated plate. General view of equations

$$
\begin{aligned}
\frac{\partial \sigma_{x}}{\partial x}+\frac{\partial \tau_{z x}}{\partial z}=0, & \frac{\partial \sigma_{z}}{\partial z}+\frac{\partial \tau_{x z}}{\partial x}=0 \\
x\left(\frac{\partial \sigma_{x z}}{\partial x}+\right. & \left.\frac{\partial \sigma_{z z}}{\partial z}\right) \\
& -z\left(\frac{\partial \sigma_{x x}}{\partial x}+\frac{\partial \sigma_{z x}}{\partial z}\right) \\
& +\sigma_{z x}-\sigma_{x z}=0 .
\end{aligned}
$$

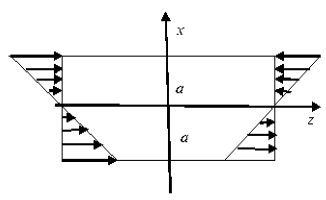

Fig. 2. Elongated plate

We will assume that there is a point $L_{1}$ where the stresses coincide. We will begin with the system of equations represents the first two equations, instead of the third equation we use the symmetry condition of the stress

$$
\begin{aligned}
& x\left(\frac{\partial \sigma_{x z}}{\partial x}+\frac{\partial \sigma_{z z}}{\partial z}\right)-z\left(\frac{\partial \sigma_{x x}}{\partial x}+\frac{\partial \sigma_{x z}}{\partial z}\right)+ \\
& \sigma_{z x}-\sigma_{x z}=0 . \text { Then }
\end{aligned}
$$

$\sigma_{z}=b_{1} x, \quad \tau_{x z}=\tau_{0}, \sigma_{x}=\tau_{01}$ is a solution to the system of equations and $\tau_{x z}=\tau_{z x}$.

Thus, an additional assumption that closes the systems of equations on the plane in classical mechanics is the condition of the stress tensor symmetry. Another option is used in work [11], the assumption about the distribution of stress. For a symmetric tensor, consider the solution: let $\sigma_{z}=$ 


$$
b(x-a)+l x+f(z) \quad \text { or } \quad \sigma_{z}=b x+
$$$$
f(z) \text {. Then }
$$

$\tau_{x z}=-\int_{0}^{x} \dot{f} d x=-\dot{f} x+F(z), \sigma_{x}{ }^{0}=$ $-\int_{L_{1}}^{z}(-\ddot{f} x+\dot{F}) d x=\ddot{f} \frac{x^{2}}{2}-\dot{F} x+M(z)$.

Let us satisfy the boundary condition $\sigma_{x}^{0}(x=a)=0$.

$\sigma_{x}^{0}=\ddot{f}(x-a)^{2} / 2-\dot{F}(x-a)$.

$\tau_{x z}=-\dot{f}(x-a)+F(z)$

Equation for moment

$x(-\dot{f}+\dot{f})-z(\ddot{f}(x-a)-\dot{F}-\ddot{f}$

$(x-a)+\dot{F})=\tau_{z x}-\tau_{x z}=\varphi(x, z)=0$.

The solution for the no symmetric tensor can be found as follows

$$
\begin{aligned}
& \frac{\partial \sigma_{x}}{\partial x}+\frac{\partial\left(\tau_{x z}+\varphi\right)}{\partial z}=0, \quad \frac{\partial \sigma_{z}}{\partial z}+\frac{\partial \tau_{x z}}{\partial x}=0 \\
& x\left(\frac{\partial \sigma_{x z}}{\partial x}+\frac{\partial \sigma_{z z}}{\partial z}\right)-z\left(\frac{\partial \sigma_{x x}}{\partial x}\right. \\
& \left.+\frac{\partial\left(\sigma_{x z}+\varphi\right)}{\partial z}\right)+\varphi=0 .
\end{aligned}
$$

At the first iteration, we consider equations only for $\varphi$, leaving the coefficients from the zero iteration. The first parenthesis vanishes. We represent the second parenthesis as

$$
\begin{aligned}
& \frac{\partial \sigma_{x x}}{\partial x}+\frac{\partial\left(\sigma_{x z}+\varphi\right)}{\partial z}=\frac{\partial \sigma_{x x}}{\partial x}+\frac{\partial \sigma_{x z}}{\partial z}+\frac{\partial \varphi}{\partial z} \\
& =0+\frac{\partial \varphi}{\partial z} \text {. Then } \\
& \frac{\partial \varphi}{\partial z}+\frac{1}{z} \varphi=0 . \quad \varphi=e^{-\int_{L_{1}}^{z} \frac{1}{z} d z}= \\
& \left.e^{-\ln \frac{1}{z}}\right|_{L_{1}} ^{z}=r z, \quad r=\text { const } \\
& \frac{\partial \varphi}{\partial z}=r z, \quad \varphi=\frac{r z^{2}}{2}+S(x) \text {. }
\end{aligned}
$$

Now you can find the corrected value of $\sigma_{x}$. In this version, the tensor will be symmetric. The tensor will not be symmetric if $f, F=0, M(z)=d(L-z)$. In this case, $\tau_{x z}=0, \quad \tau_{z x}=0$. We get the results of work

E. A. Bulanova [16]. If there are disturbances on both sides, then stitching is required at some point $L_{1}$, dependence on $x$ can be arbitrary. The influence of the moment creates a collective effect. Let us consider its effect in a rarefied gas. Fluctuations are considered as random deviations from the mean. This effect was first explained by A.Einstein. It consisted in the fact that the diffusion force should be equal to the viscous Stokes drag force

$$
\frac{\mathrm{n}_{\mathrm{p}} \mathrm{F}_{\mathrm{s}}}{\mathrm{c}_{\mathrm{s}}}=\mathrm{D}_{\mathrm{E}} \frac{\partial \mathrm{n}_{\mathrm{p}}}{\partial \mathrm{x}}
$$

Later the theory was developed on the basis of Langevin and Fokker-Planck equations [17-23]. The evolution of a Brownian particle (fluctuation) is determined by its interaction with the environment, which is always collective. In the kinetic representation, the evolution of a system of Brownian particles is described by a nonlocal equation for the n-particle distribution function. The Langevin and Fokker-Planck equations are obtained from the Liouville equation for specially selected models of integral kernels using phenomenological conservation laws. In recent years, the molecular modeling method has been widely used.

The classical Langevin equation for one particle

$\frac{d V}{d t}=-\frac{\zeta_{\ddot{\ddot{~}}}}{m} \mathrm{~V}+\frac{1}{m} F(t), \quad$ where $F(t)$ is a random force.

A Markov Gaussian process is considered with the condition that the average for an ensemble of particles $\langle F\rangle=0$. In our version, this condition is fulfilled by virtue of the fulfillment of the theorem on the conservation of the moment in a closed volume. For equilibrium condition, this is true. Langevin equation taking into account the influence of the angular momentum is $\frac{d V}{d t}=-\frac{\zeta_{\ddot{v}}}{m} \mathrm{~V}+\frac{1}{m} \frac{d M}{d r}, \quad$ where $\zeta_{\ddot{V}}$ coefficient of friction of the selected particle, $m$ is the mass of the particle, $M$ is the moment of force acting on the particle, $\mathrm{V}$ - particle velocity.

The classical Langevin equation for one particle

$\frac{d V}{d t}=-\frac{\zeta_{\ddot{i}}}{m} \mathrm{~V}+\frac{1}{m} F(t), \quad$ where $F(t)$ is a random force.

A more interesting situation arises in Coulomb plasma. The movement of the center of inertia entails a new distribution of charges. The result is a change in the strength of the electric field. In this case, the main force will be strength associated with the movement of charges.

\section{Conclusion}

Modern models of continuum mechanics do not give answers to many questions when describing 
the processes of turbulent flows, flow separation, in matters of destruction of solids, etc. It is proposed to extend the suggested model to the case of large gradients and bring the model in line with the main provisions of classical mechanics and mathematics. The necessity of using no symmetric stress tensor and a vector value of pressure in the conservation laws of continuum mechanics is substantiated. Particular examples from the theory of the boundary layer, the theory of elasticity and kinetic theory were given in previous works. We suggest an algorithm for taking into account the no symmetric stress tensor by the example of solving the problem of calculating a beam with a given distribution of one of the stresses. The ambiguity of using a symmetric stress tensor for closing and the possibility of using the results with a symmetric tensor, supplementing the solution with iteration, are shown. The proposed model can be applied to a wider range of problems in continuum mechanics than the classical theory.

\section{References}

[1]. Yu.N. Shevchenko, I. V. Prokhorenko, The theory of elastic-plastic shells under non-isothermal loading processes. Kiev. Naukova Dumka. 1981, 296.

[2]. R.A. Kayumov, Fundamentals of the theory of elasticity and elements of the theory of plates and shells: Textbook / R.A. Kayumov - Kazan: Kazan Publishing House. state architect-builds un-that. 2016, 111

[3]. P.F. Nedorezov, N.M. Sirotkina, Numerical methods for studying steady-state vibrations of viscoelastic rectangular plates and circular cylindrical shells. Saratov. Saratov University Press.

1997, 72

[4]. A.I. Koshelev, M.A. Narbut, Lectures on the mechanics of deformable solids. SPb: Publishing House of St. Petersburg University. 2003, 276.

[5].G.Z. Sharafufutdinov, Some plane problems of the theory of elasticity. Moscow: Scientific World. 2014,

[6]. A.M. Katz, Elasticity theory. St. Petersburg. 2002, 208
[7]. N.E. Kochin, I.A. Kibel, N.V. Rose, Theoretical Hydromechanics, Moscow: FizMat Literature, 1963, 583

[8].A.A. Ilyushin, Asymmetry of strain and stress tensors in the mechanics of a continuous medium. WEST. Moscow. University. Ser. 1. Mathematicsmechanics.1996. pp. 6-14

[9]. V.I. Smirnov, Higher mathematics course. T.2. M .: Nauka, 1974, 655.

[10]. E.V Prozorova . The Effect of Angular Momentum and Ostrogradsky-Gauss Theorem in the Equations of Mechanics. Wseas Transaction on fluid DOI: 10.37394/232013.2020.15.2

[11]. L.G. Loytsyansky, Mechanics of liquid and gas. M .: Nauka. 1970, 904.

[12]. Ouadie Koubaiti, Ahmed Elkhalfi, Jaouad El-Mekkaoui, Nikos Mastorakis, Solving the Problem of Constraints Due to Dirichlet Boundary Conditions in the Context of the Mini Element Method, International Journal of Mechanics, pp. 1221, Volume 14, 2020.

[13]. Tarik Chakkour, Fayssal Benkhaldoun, Slurry Pipeline for Fluid Transients in Pressurized Conduits, International Journal of Mechanics, pp. 1-11, Volume 14, 2020.

[14]. Yu.G. Stepanov, Theoretical hydrodynamics, theory of elasticity, model of a continuous medium with moment stresses. Proceedings of the Krylov State Scientific Center. 2016. Issue 93 (377). pp.163-174

[15]. N.G. Kolbasnikov, Theory of metal pressure treatment. Deformation resistance and ductility. St. Petersburg: St. Petersburg State University Publishing House, 2000, 314.

[16]. E.A. Bulanov, Moment stresses in the mechanics of a solid, bulk and liquid body. M.: University book. 2012, 140

[17]. R. Balescu. Equilibrium and nonequilibrium statistic mechanics. A Wiley-Intersciences Publication John Willey and Sons. New-YourkLondon...1975, 256

[18]. P. Resibois, M.De. Lener. Classical kinetic theory of fluids. John Wiley and Sons. New-York, London,... 1977, 423 
[19]. N.G. Van Kampen, Stochastic processes in physics and chemistry. NorthHolland. 1984, 376

[20]. Physics of Simple Liquids. Edited by H.N.V. Temperley, I.S. Rowlinson, G.S. Rushbrooke. Amsterdam, 1068, 308.

[21]. L. Boltzmann. Selected Works. Moscow: Nauka, 1984, 146

17 D.N. Zubarev, Nonequilibrium Statistical Thermodynamics, Moscow: Nauka, 1971, 416
[22]. V. Ya. Rudyak, Statistical aerohydromechanics of homogeneous and heterogeneous media. Vol.2 Novosibirsk: NGASU. 2005, 468

[23]. Yu.V. Klimontovich, Statistical theory of open systems. Vol.2, M.: Janus. K 2019, 438

\section{Creative Commons Attribution License 4.0 (Attribution 4.0 International, CC BY 4.0)}

This article is published under the terms of the Creative Commons Attribution License 4.0

https://creativecommons.org/licenses/by/4.0/deed.en_US 